\title{
BMJ Open Quality Completeness of reporting of quality improvement studies in neonatology is inadequate: a systematic literature survey
}

\author{
Zheng Jing Hu (D , ${ }^{1}$ Gerhard Fusch, ${ }^{2}$ Catherine $\mathrm{Hu},{ }^{3}$ Jie Yi Wang, ${ }^{4}$ Zoe el Helou, ${ }^{5}$ \\ Muhammad Taaha Hassan, ${ }^{5}$ Lawrence Mbuagbaw (D) , ${ }^{1}$ Salhab el Helou, ${ }^{2}$ \\ Lehana Thabane $^{1}$
}

To cite: Hu ZJ, Fusch G, Hu C, et al. Completeness of reporting of quality improvement studies in neonatology is inadequate: a systematic literature survey. BMJ Open Quality 2021;10:e001273. doi:10.1136/ bmjoq-2020-001273

- Additional supplemental material is published online only. To view, please visit the journal online (http://dx.doi.org/10. 1136/bmjoq-2020-001273).

$\mathrm{CH}, \mathrm{JYW}$, ZeH and MTH contributed equally.

Received 9 November 2020 Accepted 2 June 2021
Check for updates

(c) Author(s) (or their employer(s)) 2021. Re-use permitted under CC BY-NC. No commercial re-use. See rights and permissions. Published by BMJ.

For numbered affiliations see end of article.

Correspondence to Dr Lehana Thabane; thaban!@mcmaster.ca

\section{ABSTRACT}

Introduction Quality improvement (QI) is a growing field of inquiry in healthcare, but the reporting quality of QI studies in neonatology remains unclear. We conducted a systematic survey of the literature to assess the reporting quality of QI studies and factors associated with reporting quality.

Methods We searched Medline for publications of Ql studies from 2016 to 16 April 2020. Pairs of reviewers independently screened citations and assessed reporting quality using a 31-item modified Standards for Quality Improvement Reporting Excellence, 2nd edition (SQUIRE 2.0) checklist. We reported the number (percentage) of studies that reported each item and their corresponding 95\% Cls. We used Poisson regression to explore factors associated with reporting quality, namely, journal endorsement of SQUIRE 2.0, declaration of funding sources, year of publication and number of authors. The results were reported as incidence rate ratio (IRR) and $95 \% \mathrm{Cl}$.

Results of 1921 citations, 336 were eligible; among them, we randomly selected 100 articles to assess reporting quality. The mean (standard deviation) number of SQUIRE 2.0 items adhered to was 22.0 (4.5). Percentage of articles reporting each item varied from $26 \%$ to $100 \%$. Journal endorsement of SQUIRE 2.0 (IRR=1.11, 95\% Cl 1.02 to $1.21, p=0.015)$, declaration of funding sources and increasing number of authors were significantly associated with better reporting.

Conclusions Reporting quality of $\mathrm{Q}$ studies in neonatology is inadequate. Endorsing the SQUIRE 2.0 guideline is a step that journals can implement to enhance the completeness of reporting.

\section{INTRODUCTION}

Quality improvement (QI) efforts in healthcare have become an increasingly active field of inquiry. QI efforts have been implemented in various health settings with diverse aims, including reducing medical errors, improving patient safety, providing better satisfaction with care, increasing efficiency of healthcare delivery, or training healthcare practitioners to adhere to evidence-based practices. ${ }^{12} \mathrm{QI}$ projects that are reported clearly and rigorously can provide clear evidence of effective activities for improving the quality of care at local health settings, and thus accelerate the dissemination and adaptation of these effective practices.

The current reporting quality guideline for QI studies is the Standards for Quality Improvement Reporting Excellence, 2nd edition (SQUIRE 2.0), published in 2015. SQUIRE 2.0 is intended for any study that report on systematic, data-driven efforts to improve the quality, safety and value of healthcare. SQUIRE 2.0 was developed as an improvement to SQUIRE 1.0, to provide better guidance for authors in writing more clearly, precisely, completely and transparently about QI studies. ${ }^{3}$

Commonly used reporting guidelines such as the Consolidated Standards of Reporting Trials (CONSORT), The Strengthening the Reporting of Observational Studies in Epidemiology or Preferred Reporting Items for Systematic Reviews and Meta-Analyses are intended for specific study designs (ie, randomised controlled trials (RCTs), observational studies and systematic reviews). ${ }^{4}$ In contrast, the applicability of SQUIRE 2.0 depends on a study's objectives, rather than its design; namely, that the study sought to report on a systematic effort to improve the quality, safety and value of healthcare at a systems-level. For example, a study that reported on a systematic effort to reduce nosocomial sepsis qualifies for SQUIRE 2.0 evaluation. However, a study that aimed to assess risk factors of nosocomial sepsis, or investigate a specific treatment regimen, would not qualify for SQUIRE 2.0 evaluation, because a systematic improvement effort was absent. In this context, QI studies undoubtedly encompass a diverse range of study designs and methodologies. 
Thus, a significant challenge to achieving clear and consistent reporting of QI studies is the large variation in how QI research is conducted and the definition of QI itself. ${ }^{5-8}$ QI studies may vary in intervention methodology, such as iterative Plan-Do-Study-Act cycles, Lean six sigma and Total Quality management. QI studies may also differ in study objectives, such as evaluate the success and feasibility implementing evidence-based practice into a local setting, reduce adverse events, or improve healthcare workers' well-being. Each of these study designs and objectives may have their own reporting practices and cater to different stakeholders' priorities. Consequently, one would anticipate a large variation in reporting, and challenges in achieving clarity and consistency when these studies are reported in the general healthcare improvement context. Woods and Martin describe 'insufficient attention to rigorous evaluation of improvement and to sharing the lessons of successes and failures ${ }^{, 9}$ as a critical barrier to the effectiveness of QI. Altogether, these realities emphasise the need to develop evidencebased inquiry in this field and assess the completeness of reporting of QI studies as part of this process.

A previous study by Howell et al showed that the quality of reporting of QI studies did not demonstrate improvement following the publication of SQUIRE 1.0. ${ }^{10}$ Otherwise, to our best knowledge, there have not been any studies that evaluated the completeness of reporting of QI studies using SQUIRE 2.0 in the neonatology literature.

In a Neonatal Intensive Care Unit environment, unsafe care such as medication administration errors or inconsistencies in the quality and key processes of care, can lead to neonates' adverse outcomes that incur long-term developmental consequences. ${ }^{1}$ QI efforts have been shown to improve outcomes in neonatal care. ${ }^{2}$ Thus, clear reporting of these efforts is paramount to facilitate knowledge translation and accelerate progress on the safety and quality of neonatal care.

This literature survey aims to inform clinicians and researchers in neonatology on the current state of reporting quality of QI studies. The primary objective is to assess the published studies' compliance with SQUIRE 2.0. The secondary objective is to identify the characteristics of published articles associated with their quality of reporting.

\section{METHODS}

This study was a systematic survey of the literature. In a systematic survey, the literature review is conducted on a random sample of all eligible articles retrieved from a search strategy; whereby the sampling strategy is determined a-priori. Furthermore, the search strategy aims at retrieving a sufficient sample of articles that reflect the research question being addressed. ${ }^{11}$ We searched the Medline database for publications from 2016 to 16 April 2020, as defined by the "Year of Publication" field using the search strategy shown in online supplemental appendix A1. Search terms consisted of Medical Subject Headings
(MeSH) keywords pertaining to quality improvement and neonatology. These terms were determined with a librarian and based on previous studies of QI search strategies. ${ }^{12}$ The search aimed to find QI studies published after the release of SQUIRE 2.0 in September 2015. ${ }^{13}$

The primary outcome was the overall quality of reporting, as measured by the number of items that published papers adhered to in a modified SQUIRE 2.0 checklist. In addition, we reported the percentage of studies that adhered to each checklist item along with 95\% CIs. We modified the SQUIRE 2.0 checklist for our assessment following a pilot testing of SQUIRE 2.0 with 10 articles and discussion with QI expert $\mathrm{SeH}$. Online supplemental appendix A2 details the corresponding SQUIRE 2.0 statement of these items and reasons for modifying them. Thus, this checklist consists of 31 items.

Herein, we modified the SQUIRE 2.0 checklist for our three main reasons. First, the title was excluded because it was considered as inclusion criteria. Second, the abstract was excluded as well because all included studies had an abstract. Third, some items were not universally applicable to all QI reports, and as such, were excluded from our quality of reporting assessment. Finally, reporting items that expressed similar ideas were combined, while single reporting items that consisted of multiple ideas were split into separate items. We made these decisions based on the details of the items' explanation and elaboration, and after pilot-testing the SQUIRE 2.0 checklist on 10 articles. The developers SQUIRE 2.0 themselves also stated that "...some (SQUIRE 2.0) items may not be relevant for inclusion in a particular manuscript ". ${ }^{3}$ Therefore, we used pilot testing and clinical rationale to decide which items were universally applicable to all QI reports. See online supplemental appendix A2 for a detailed table of specific items modified, our rationale for modifying them, and the implications of these modifications.

We defined QI publications as studies whose primary objective was to test interventions that lead to better patient outcomes, stronger system performance or enhanced professional development. Our exclusion criteria consisted of literature reviews, study protocols, articles not written in English, editorial commentary and studies whose primary focus was not on healthcare improvement. We implemented a two-stage title/abstract screening process due to documented challenges of defining QI and selecting appropriate studies for a QI literature review. ${ }^{5}$

In stage 1, we included all single studies that consisted of an intervention, primary outcome, and a description of how outcomes changed over time, and all studies that explicitly declared their study as a 'quality improvement' or 'quality initiative' effort in the title or abstract. Two pairs of student reviewers $\mathrm{ZeH}$ and $\mathrm{MTH}$, and $\mathrm{CH}$ and JYW) screened the title, abstract, and full text where necessary, and disagreements between two reviewers were resolved by ZJH. Online supplemental appendix A4, box 1 provides details on the decision process for determining the inclusion of articles. 


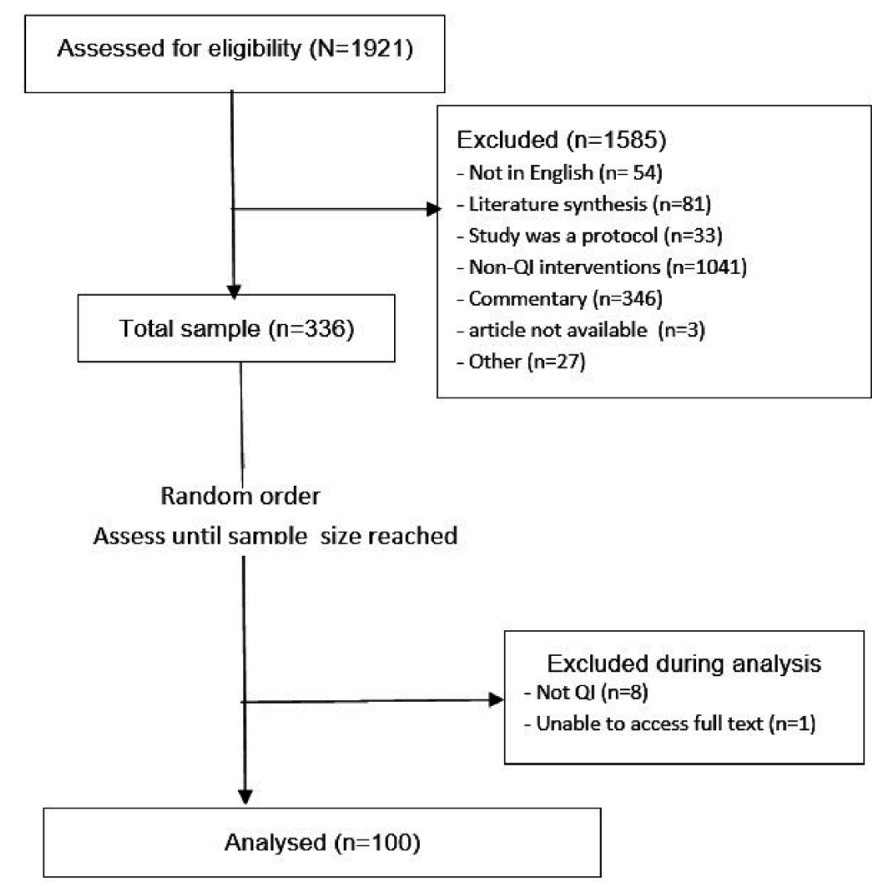

Figure 1 Flow diagram describing the articles' selection process. QI, quality improvement.

Subsequently, the title/abstract of all selected articles were rescreened to determine whether the authors intended to publish their studies as a healthcare improvement effort. Here, we included studies that described intervention(s) to improve a specific process, quality or safety of care. ZJH and QI experts SeH and GF assessed each article's abstract and full-text and reached a consensus on selecting articles for data abstraction. Online supplemental appendix A3 provides a list of attributes that the assessors examined when evaluating a study's eligibility for data abstraction.

We stratified all successfully screened articles by year of publication. Within each stratum, the articles were first sorted randomly using Excel. Subsequently, each article was sampled without replacement in their sorted order, according to the probability that an article belonged to a specific stratum (year). Using this process, we ordered all articles randomly and assessed them in this order until we reached the desired sample size.

We conducted data abstraction on the modified SQUIRE 2.0 checklist. Ten articles were assessed during the initial pilot testing. Each reviewer assessed four articles, and ZJH assessed all 10 articles and compared results. We resolved disagreements through discussion. Subsequent articles were divided between the four student reviewers and assessed independently with ZJH. Disagreements were resolved through discussion, and with a third author (SeH or GF) if needed.

We examined explanatory variables to determine factors associated with the quality of reporting. We determined these factors a-priori based on evidence from previous literature that assessed reporting quality. These factors include: endorsement of SQUIRE 2.0 by the journal, defined as the presence of recommendation or requirement to comply with SQUIRE 2.0 among instructions for authors (ie, Journal endorsement of reporting guideline),${ }^{13-16}$ declaration of funding source, defined as both the presence of a funding section and a declaration of a specific organisation that provided funding, ${ }^{17} 18$ year of publication (implying more recent studies have a better quality of reporting), ${ }^{19-21}$ and the number of authors listed on a manuscript, excluding organisations or groups as authors. ${ }^{172122}$

\section{Analysis}

We summarised the characteristics of the included studies using descriptive statistics with categorical variables reported as frequencies. We summarised continuous variables using median and IQR or mean (SD) where appropriate. We computed the proportion of articles that reported each item, along with their 95\% CIs; and we calculated 95\% CIs using the Wilson Score method. ${ }^{23}$ To explore factors associated with reporting quality, we fitted a Poisson model assuming an identity link to the data for both univariable and multivariable analysis. The incidence rate ratio (IRR) for each factor was reported with $95 \%$ CIs and $p$ values. We checked the Poisson model's assumptions by examining the dispersion parameters. We assessed collinearity by examining the correlation matrix between all variables, condition index number and variance inflation factor.

Finally, to explore interrater agreement, we computed Cohen's Kappa ${ }^{24}$ for a selected list of SQUIRE 2.0 items. We chose to assess agreement for items that had a more subjective interpretation, where reporting the extent of agreement would be informative. We also chose three items with more objective interpretation as a counterbalance. We excluded data for 26 articles in the agreement assessment, as one reviewer had a particularly low preconsensus agreement. All analyses were conducted using Microsoft Excel and SAS V.9.4.

\section{Sample size}

The aim of sample size calculation in this study was to estimate the proportion of articles that reported each item in the modified SQUIRE 2.0 checklist, in line with numerous previous studies that assessed the completeness of reporting. ${ }^{25-27}$ Thus, we estimated sample size using the population proportion CI method. ${ }^{28}$ The desired margin of error was $10 \%$, and the estimated population proportion was 0.50 based on the conservative approach. Using this approach, we calculated a sample size of 97 . As we could assess additional articles, 100 articles were evaluated.

\section{Patient and public involvement}

Patients and the public were not involved in the design, or conduct, or reporting, or dissemination plans of our research.

\section{RESULTS}

Our search retrieved 1921 articles. After the selection process, 336 articles remained available for data 
Table 2 Frequency of reporting for each item in the modified SQUIRE 2.0 checklist

\section{SQUIRE 2.0 item}

Frequency

Proportion (95\% Cl)

Background

Nature and significance of the local problem

100

1.00 (0.96 to 1.00$)$

Summary of what is currently known about the problem, including relevant

98

0.98 (0.93 to 0.99$)$

previous studies

Informal or formal frameworks, models, concepts, and/or theories used to

80

$0.80(0.71$ to 0.87$)$

explain the problem, any reasons or assumptions that were used to develop the

intervention(s), and reasons why the intervention(s) was expected to work

Purpose of the project and of this report

93

$0.93(0.86$ to 0.97$)$

\section{Methods}

Contextual elements considered important at the outset of introducing the intervention(s)

$92 \quad 0.92(0.85$ to 0.96$)$

Description of the intervention(s) in sufficient detail that others could reproduce it 96

$0.96(0.90$ to 0.98$)$

Specifics of the team involved in the work

$88 \quad 0.88(0.80$ to 0.93$)$

Approach chosen for assessing the impact of the intervention(s)

86

0.86 (0.78 to 0.91$)$

Measures chosen for studying processes and outcomes of the intervention(s),

including rationale for choosing them, their operational definitions, and their

validity and reliability (outcome)

Measures chosen for studying processes and outcomes of the intervention(s), including rationale for choosing them, their operational definitions, and their validity and reliability (process)

Description of the approach to the ongoing assessment of contextual elements that contributed to the success, failure, efficiency, and cost

$82 \quad 0.82(0.73$ to 0.88$)$

Qualitative and quantitative methods used to draw inferences from the data

$0.49(0.39$ to 0.59$)$

Methods for understanding variation within the data, including the effects of time as a variable

Methods employed for assessing completeness and accuracy of data

$0.63(0.53$ to 0.72$)$

Ethical aspects of implementing and studying the intervention(s) and how they were addressed, including, but not limited to, formal ethics review and potential conflict(s) of interest

\section{Results}

Initial steps of the intervention(s) and their evolution over time (eg, time-line diagram, flow chart or table), including modifications made to the intervention during the project

Details of the process measures and outcome (outcome)

97

0.97 (0.92 to 0.99$)$

Details of the process measures and outcome (process)

73

0.73 (0.64 to 0.81$)$

Contextual elements that interacted with the intervention(s)

29

0.29 (0.21 to 0.39$)$

Unintended consequences such as unexpected benefits, problems, failures, or costs associated with the intervention(s).

Details about missing data

0.43 (0.34 to 0.53$)$

\section{Discussion and conclusion}

Key findings, including relevance to the rationale and specific aims

26

$0.26(0.18$ to 0.35$)$

Particular strengths of the project

$\begin{array}{ll}97 & 0.97(0.92 \text { to } 0.99) \\ 60 & 0.60(0.50 \text { to } 0.69) \\ 83 & 0.83(0.74 \text { to } 0.89) \\ 80 & 0.80(0.71 \text { to } 0.87) \\ 50 & 0.50(0.40 \text { to } 0.60) \\ 59 & 0.59(0.49 \text { to } 0.68)\end{array}$

Nature of the association between the intervention(s) and the outcomes

Continued

Comparison of results with findings from other publications Impact of project on people and systems

Reasons for any differences between observed and anticipated outcomes, including the influence of context 


\section{Table 2 Continued}

\section{SQUIRE 2.0 item}

Frequency

92

1. Limits to the generalisability of the work

2. Factors that might have limited internal validity such as confounding, bias, or imprecision in the design, methods, measurement, or analysis

Efforts made to minimise and adjust for limitations
Usefulness of the work

Sustainability

SQUIRE, Standa

SQUIRE, Standards for Quality Improvement Reporting Excellence.

absolute and relative effect sizes if the outcome measure is binary. ${ }^{33}$ Similar 'loose requirements' can be found for numerous items in SQUIRE 2.0.

Thus, a critical methodological limitation of using SQUIRE 2.0 for assessing reporting quality is the absence and subjectivity of information on how much details should be described for specific important items to satisfy the reporting criteria. Consequently, assessment of reporting quality can differ considerably between reviewers. Furthermore, some poorly written articles can still receive a high SQUIRE 2.0 score. A second limitation is the difficulty of assessing the presence of QI expertise in an authorship team. Practitioners who conduct QI projects specialise in diverse academic disciplines, and may lack a comprehensive understanding of QI. Thus, one would anticipate that the presence of a QI expert in the authorship team would improve reporting or ensure that the manuscript is more QI-focused. However, ascertaining whether an author is a QI expert may involve an extensive web search of their institutional affiliations, publication activities and curriculum vitae (if available online). Furthermore, the manuscript may not indicate the extent of involvement of the QI expert in the study. Hence, we could not assess authors' QI expertise as a factor for influencing the quality of reporting.

The subjective description of SQUIRE 2.0 items also impeded inter-rater reliability. In this study, preconsensus agreement between reviewers was poor. The Kappa statistic for various selected items ranged from 0 to 0.64 . In comparison, previous RCT reporting quality studies on emergency medicine, brain tumour RCTs, and heart failure showed Kappa ranging from 0.80 to $0.90 .^{18} 2634$ However, even among QI experts, agreement on whether published papers met specific criteria was suboptimal, with a Kappa of $0.52 .^{35}$

We examined post-hoc whether published studies primarily adhered to other reporting guidelines, and if this may have affected their adherence to SQUIRE 2.0. However, the results show that only one study adhered to a guideline besides SQUIRE 2.0. Most studies did not indicate following any reporting guideline and the rest adhered to SQUIRE 2.0 itself. Thus, we were unable to make any conclusions in this regard.

Presently, journals should endorse the SQUIRE 2.0 guideline by recommending its use or mandating its adherence to improve the completeness of reporting of QI studies. Nonetheless, improving published studies' adherence to SQUIRE 2.0 alone is not sufficient for publishing well-written QI reports. Ultimately, SQUIRE 2.0 was intended to provide general reporting guidance for authors who are interested in publishing their QI efforts. As such, SQUIRE 2.0 has limitations for assessing important methodological aspects of reporting regarding specific QI methodologies. Future work can include conducting a critical appraisal of QI publications, assessing the reporting of the interventions themselves, or assessing the association between SQUIRE 2.0 adherence, requirement, and endorsement on the quality of QI evidence. Both assessments would provide valuable insights into the reporting of methodological aspects of QI studies in neonatology.

Table 3 Univariable and multivariable analysis of factors associated with the number of items reported in each article

\begin{tabular}{|c|c|c|c|c|c|}
\hline \multirow[b]{2}{*}{ Attribute } & \multirow[b]{2}{*}{ Category } & \multicolumn{2}{|l|}{ Univariable analysis } & \multicolumn{2}{|l|}{ Multivariable analysis } \\
\hline & & $\begin{array}{l}\text { Incidence rate ratio } \\
(95 \% \mathrm{Cl})\end{array}$ & $P$ value & $\begin{array}{l}\text { Incidence rate ratio } \\
(95 \% \mathrm{Cl})\end{array}$ & $P$ value \\
\hline Journal endorses SQUIRE 2.0 & No & REF & & REF & \\
\hline Declared funding sources & Yes & 1.14 (1.04 to 1.24$)$ & 0.003 & 1.11 (1.02 to 1.21$)$ & 0.021 \\
\hline Number of authors & & $1.02(1.01$ to 1.04$)$ & 0.001 & 1.02 (1.01 to 1.03$)$ & 0.003 \\
\hline
\end{tabular}

SQUIRE, Standards for Quality Improvement Reporting Excellence; REF, reference level. 
Table 4 Assessment of reporting agreement on SQUIRE items, preconsensus

\begin{tabular}{|c|c|c|c|}
\hline Items & $\begin{array}{l}\text { Percent } \\
\text { Agreement }\end{array}$ & Kappa & 95\% Cl (Kappa) \\
\hline $\begin{array}{l}\text { Rationale: What Ql framework, model, concept, or theory is } \\
\text { being used to address the problem? }\end{array}$ & 0.63 & 0.02 & -0.21 to 0.25 \\
\hline Description of QI team involved & 0.79 & 0.32 & 0.06 to 0.59 \\
\hline $\begin{array}{l}\text { Qualitative and quantitative methods used to draw inferences } \\
\text { from the data }\end{array}$ & 0.69 & 0.31 & 0.09 to 0.54 \\
\hline $\begin{array}{l}\text { Methods for understanding variation within the data, including } \\
\text { the effects of time as a variable }\end{array}$ & 0.68 & 0.36 & 0.13 to 0.57 \\
\hline Process measures stated & 0.67 & 0.32 & 0.11 to 0.53 \\
\hline $\begin{array}{l}\text { Measures for assessing contextual factors (that contributed to } \\
\text { the success, failure, efficiency or cost of intervention) }\end{array}$ & 0.54 & 0.07 & -0.14 to 0.29 \\
\hline $\begin{array}{l}\text { Initial steps of the intervention(s) and their evolution over } \\
\text { time (eg, time-line diagram, flow chart or table), including } \\
\text { modifications made to the intervention during the project }\end{array}$ & 0.82 & 0.64 & 0.47 to 0.82 \\
\hline Process measures evaluated & 0.65 & 0.21 & -0.02 to 0.45 \\
\hline Contextual factors analysed & 0.81 & 0.39 & 0.13 to 0.64 \\
\hline Limitations to internal and external validity & 0.92 & 0.58 & 0.28 to 0.88 \\
\hline Impact of project on people and systems & 0.61 & 0.21 & -0.02 to 0.43 \\
\hline Usefulness of work & 0.63 & 0.17 & -0.02 to 0.35 \\
\hline
\end{tabular}

*26 articles removed due to low agreement with one individual.

QI, quality improvement.

\section{CONCLUSION}

Overall, the quality of reporting of QI studies in neonatology is inadequate. Although SQUIRE 2.0 serves as a suitable guideline for reporting QI efforts clearly, its ability to assess reporting of key methodological details is limited. Future studies examining the reporting of QI methodologies, or the relationship between SQUIRE 2.0 adherence and strength of evidence, would inform a better understanding of QI reporting and how it can be improved.

\section{Author affiliations}

${ }^{1}$ Department of Health Research Methods Evidence and Impact, McMaster University, Hamilton, Ontario, Canada

${ }^{2}$ Division of Neonatology, Department of Pediatrics, McMaster University, Hamilton, Ontario, Canada

${ }^{3}$ Bachelor of Arts and Science, McMaster University, Hamilton, Ontario, Canada ${ }^{4}$ Bachelor of Medical Sciences, Schulich School of Medicine \& Dentistry, University of Western Ontario, London, Ontario, Canada

${ }^{5}$ Bachelor of Health Sciences, Faculty of Health Sciences, McMaster University, Hamilton, Ontario, Canada

Contributors ZJH drafted the manuscript and performed statistical analysis. GF and SeH contributed to the development of the selection criteria, data extraction criteria and search strategy. ZJH, CH, JYW, MTH and ZeH performed data abstraction, while SeH and GF provided expertise on the SQUIRE 2.0 guideline when needed. LM provided suggestions to improve the manuscript. LT is the guarantor of the review and provided expertise on quality of reporting, manuscript writing, and statistical analysis. All authors read, provided feedback and approved the final manuscript.

Funding The authors have not declared a specific grant for this research from any funding agency in the public, commercial or not-for-profit sectors.

Competing interests None declared.

Patient consent for publication Not required.
Provenance and peer review Not commissioned; externally peer reviewed.

Supplemental material This content has been supplied by the author(s). It has not been vetted by BMJ Publishing Group Limited (BMJ) and may not have been peer-reviewed. Any opinions or recommendations discussed are solely those of the author(s) and are not endorsed by BMJ. BMJ disclaims all liability and responsibility arising from any reliance placed on the content. Where the content includes any translated material, BMJ does not warrant the accuracy and reliability of the translations (including but not limited to local regulations, clinical guidelines, terminology, drug names and drug dosages), and is not responsible for any error and/or omissions arising from translation and adaptation or otherwise.

Open access This is an open access article distributed in accordance with the Creative Commons Attribution Non Commercial (CC BY-NC 4.0) license, which permits others to distribute, remix, adapt, build upon this work non-commercially, and license their derivative works on different terms, provided the original work is properly cited, appropriate credit is given, any changes made indicated, and the use is non-commercial. See: http://creativecommons.org/licenses/by-nc/4.0/.

ORCID iDs

Zheng Jing Hu http://orcid.org/0000-0002-2215-3353

Lawrence Mbuagbaw http://orcid.org/0000-0001-5855-5461

\section{REFERENCES}

1 Lachman P, Jayadev A, Rahi M. The case for quality improvement in the neonatal intensive care unit. Early Hum Dev 2014;90:719-23.

2 Ellsbury DL, Clark RH. Does quality improvement work in neonatology improve clinical outcomes? Curr Opin Pediatr 2017;29:129-34.

3 Goodman D, Ogrinc G, Davies L, et al. Explanation and elaboration of the Squire (standards for quality improvement reporting excellence) guidelines, V.2.0: examples of Squire elements in the healthcare improvement literature. BMJ Qual Saf 2016;25:e7.

4 Ogrinc G, Davies L, Goodman D, et al. Squire 2.0 (standards for quality improvement reporting excellence): revised publication guidelines from a detailed consensus process. BMJ Qual Saf 2016;25:986-92.

5 Jones E, Furnival J, Carter W. Identifying and resolving the frustrations of reviewing the improvement literature: the experiences of two improvement researchers. BMJ Open Qual 2019;8:e000701. 
6 Jones EL, Dixon-Woods M, Martin GP. Why is reporting quality improvement so hard? A qualitative study in perioperative care. $B M J$ Open 2019;9:e030269.

7 Leviton L. Reconciling complexity and classification in quality improvement research. BMJ Qual Saf 2011;20(Suppl 1):i28-9.

8 BMJ Quality \& Safety. How to study improvement interventions: a brief overview of possible study types. Available: https:// qualitysafety-bmj-com.libaccess.lib.mcmaster.ca/content/24/5/325 [Accessed 13 Sep 2020].

9 Dixon-Woods M, Martin GP. Does quality improvement improve quality? Future Hosp J 2016;3:191-4.

10 Howell V, Schwartz AE, O'Leary JD, et al. The effect of the Squire (standards of quality improvement reporting excellence) guidelines on reporting standards in the quality improvement literature: a before-and-after study. BMJ Qual Saf 2015;24:400-6.

11 Mbuagbaw L, Lawson DO, Puljak L, et al. A tutorial on methodological studies: the what, when, how and why. BMC Med Res Methodol 2020;20:226.

12 Hempel S, Rubenstein LV, Shanman RM, et al. Identifying quality improvement intervention publications--a comparison of electronic search strategies. Implement Sci 2011;6:85.

13 Stevens A, Shamseer L, Weinstein E, et al. Relation of completeness of reporting of health research to journals endorsement of reporting guidelines: systematic review. BMJ 2014;348:g3804.

14 Panic N, Leoncini E, de Belvis G, et al. Evaluation of the endorsement of the preferred reporting items for systematic reviews and meta-analysis (PRISMA) statement on the quality of published systematic review and meta-analyses. PLoS One 2013;8:e83138.

15 Liu LQ, Morris PJ, Pengel LHM. Compliance to the CONSORT statement of randomized controlled trials in solid organ transplantation: a 3-year overview. Transpl Int 2013;26:300-6.

16 Ethgen M, Boutron L, Steg PG, et al. Quality of reporting internal and external validity data from randomized controlled trials evaluating stents for percutaneous coronary intervention. BMC Med Res Methodol 2009;9:24.

17 Balasubramanian SP, Wiener M, Alshameeri Z, et al. Standards of reporting of randomized controlled trials in general surgery: can we do better? Ann Surg 2006;244:663-7.

18 Borg Debono V, Zhang S, Ye C, et al. The quality of reporting of RCTs used within a postoperative pain management meta-analysis, using the CONSORT statement. BMC Anesthesiol 2012;12:13.

19 Chowers MY, Gottesman BS, Leibovici L, et al. Reporting of adverse events in randomized controlled trials of highly active antiretroviral therapy: systematic review. J Antimicrob Chemother 2009;64:239-50.
20 Farrokhyar F, Chu R, Whitlock R, et al. A systematic review of the quality of publications reporting coronary artery bypass grafting trials. Can J Surg 2007;50:266-77.

21 Zheng SL, Chan FT, Maclean E, et al. Reporting trends of randomised controlled trials in heart failure with preserved ejection fraction: a systematic review. Open Heart 2016;3:e000449.

22 Yao AC, Khajuria A, Camm CF, et al. The reporting quality of parallel randomised controlled trials in ophthalmic surgery in 2011: a systematic review. Eye 2014;28:1341-9.

23 7.2.4.1. confidence intervals. Available: https://www.itl.nist.gov/ div898/handbook/prc/section2/prc241.htm [Accessed 26 Sep 2020].

$24 \mathrm{McHugh}$ ML. Interrater reliability: the kappa statistic. Biochem Med 2012;22:276-82.

25 Shanthanna H, Kaushal A, Mbuagbaw L, et al. A cross-sectional study of the reporting quality of pilot or feasibility trials in high-impact anesthesia journals. Can J Anaesth 2018;65:1180-95.

26 Lai R, Chu R, Fraumeni M, et al. Quality of randomized controlled trials reporting in the primary treatment of brain tumors. J Clin Oncol 2006;24:1136-44.

27 Isiguzo GC, Zunza M, Chirehwa M, et al. Quality of pilot trial Abstracts in heart failure is suboptimal: a systematic survey. Pilot Feasibility Stud 2018;4:107.

28 5.3.3 - Sample Size Computation ISTAT 500. PennState: Statistics Online Courses. Available: https://online.stat.psu.edu/stat500/lesson/ 5/5.3/5.3.3 [Accessed 29 Mar 2021].

29 Kosa SD, Monize J, Leenus A, et al. Reporting quality of pilot clinical trials in chronic kidney disease patients on hemodialysis: a methodological survey. Pilot Feasibility Stud 2019;5:53.

30 Thabane L, Thomas T, Ye C, et al. Posing the research question: not so simple. J Can Anesth 2009;56:71-9.

31 Adie S, Harris IA, Naylor JM, et al. Consort compliance in surgical randomized trials: are we there yet? A systematic review. Ann Surg 2013;258:872-8.

32 Marshman Z, Farid F. The quality of reporting of randomised controlled trials in dental public health. Community Dent Health 2010;27:253-6.

33 Moher D, Hopewell S, Schulz KF, et al. Consort 2010 explanation and elaboration: updated guidelines for reporting parallel group randomised trials. Int J Surg 2012;10:28-55.

34 Germini F, Marcucci M, Fedele M, et al. Quality of reporting in Abstracts of RCTs published in emergency medicine journals: a systematic survey of the literature suggests we can do better. Emerg Med J 2019:660-5.

35 Hempel S, Shekelle PG, Liu JL, et al. Development of the quality improvement minimum quality criteria set (QI-MQCS): a tool for critical appraisal of quality improvement intervention publications. BMJ Qual Saf 2015;24:796-804. 